\title{
Reflexões sobre as recomendações para prevenção de lesões por pressão durante a pandemia de COVID-19
}

\section{Reflections on recommendations for the prevention of pressure injuries during the COVID-19 pandemic}

\section{Reflexiones sobre las recomendaciones para la prevención de lesiones por presión durante la pandemia de COVID-19}

\author{
Aline de Oliveira Ramalho, ${ }^{1,2}$, Paula de Souza Silva Freitas ${ }^{3}$, Juliano Teixeira Moraes 4 , \\ Paula Cristina Nogueira ${ }^{5}$
}

ORCID IDS

Ramalho AO (D) https://orcid.org/0000-0001-6065-5488

Freitas PSS (D) https://orcid.org/0000-0001-9066-3286

Moraes JT (iD) https://orcid.org/0000-0002-1109-962X

Nogueira PC (D) https://orcid.org/0000-0001-5200-1281

\section{COMO CITAR}

Ramalho AO; Freitas PSS; Moraes JT; Nogueira PC. Reflexões sobre as recomendações para prevenção de lesões por pressão durante a pandemia de covid-19. ESTIMA, Braz. J. Enterostomal Ther., 2020, 18: e2520. https://doi.org/10.30886/estima.v18.940_PT

\section{RESUMO}

Objetivo: refletir sobre as recomendações específicas para prevenção de lesão por pressão em pacientes acometidos pelo novo coronavírus (COVID-19) em terapia intensiva. Método: estudo do tipo reflexão teórica fundamentada no conceito de lesão por pressão definido pelo National Pressure Injury Advisory Panel. Resultados: os conteúdos foram categorizados em três eixos temáticos: fatores de risco de desenvolvimento de lesão por pressão específicos para o paciente com COVID-19; recomendações internacionais sobre lesão por pressão em pacientes com COVID-19 e desafios para implementação das recomendações de prevenção de lesão por pressão frente à pandemia de COVID-19 no cenário brasileiro. Conclusão: para promover a prevenção de lesão por pressão em pacientes de terapia intensiva com COVID-19 é fundamental que os profissionais de saúde sejam capazes de implementar intervenções avançadas, de forma a mitigar os riscos intrínsecos e extrínsecos específicos desse paciente. No entanto, é imprescindível reconhecer as lesões potencialmente evitáveis, diferenciando-as das possíveis inevitáveis, bem como a realização de diagnóstico diferencial de lesões decorrentes da própria patogenia do vírus.

DESCRITORES: Lesão por pressão; Infecções por coronavirus; Cuidados críticos; Ferimentos e lesões; Enfermagem; Estomaterapia.

\footnotetext{
1. Universidade de São Paulo - Escola de Enfermagem - Programa de Pós-Graduação em Enfermagem na Saúde do Adulto São Paulo (SP), Brasil.

2. Hospital Sírio Libanês - Enfermagem Especializada - Estomaterapia - São Paulo (SP), Brasil.

3. Universidade Federal do Espírito Santo - Departamento de Enfermagem - Programa de Pós-Graduação em Enfermagem Vitória (ES), Brasil.

4. Universidade Federal de São João del-Rei - Programa de Pós-Graduação em Enfermagem - Divinópolis (MG), Brasil.

5. Universidade de São Paulo - Escola de Enfermagem - Departamento de Enfermagem Médico-Cirúrgica - São Paulo (SP), Brasil.

*Autora correspondente: alineo_ramalho@hotmail.com
}

Recebido: Ago. 21, 2020 | Aceito: Out. 15, 2020 


\begin{abstract}
Objective: To reflect on specific recommendations for the prevention of pressure injuries in patients affected by the new coronavirus (COVID-19) in intensive care. Method: theoretical reflection study based on the concept of pressure injury defined by the National Pressure Injury Advisory Panel. Results: the contents were categorized into three thematic axes: specific risk factors for the development of pressure injuries for patients with COVID-19; international recommendations on pressure injuries in patients with COVID-19 and challenges for implementing recommendations for preventing pressure injuries in the face of the COVID-19 pandemic in the Brazilian scenario. Conclusion: to promote the prevention of pressure injuries in intensive care patients with COVID-19 it is essential that health professionals are able to implement advanced interventions, in order to mitigate the intrinsic and extrinsic risks specific to that patient. However, it is essential to recognize potentially preventable lesions, differentiating them from possible inevitable ones, as well as making a differential diagnosis of lesions resulting from the pathogenesis of the virus itself.
\end{abstract}

DESCRIPTORS: Pressure injury; Coronavirus infections; Critical care; Wounds and injuries; Nursing; Stomatherapy.

\title{
RESUMEN
}

Objetivo: reflexionar sobre la prevención de lesiones por presión (LP) para pacientes con COVID-19 en cuidados intensivos. Método: ensayo teórico-reflexivo a la luz de las recomendaciones establecidas por el National Pressure Injury Advisory Panel. Resultados: Después de recopilar los materiales publicados, los contenidos se clasificaron en tres ejes temáticos: factores de riesgo para desarrollar LP específicos para el paciente con COVID-19; recomendaciones internacionales sobre LP en pacientes con COVID-19 y desafíos para implementar recomendaciones de prevención de LP en la pandemia de COVID-19 en el escenario brasileño. Conclusión: Para prevenir la LP en pacientes de cuidados intensivos con COVID-19, es esencial que los profesionales de la salud puedan implementar intervenciones avanzadas, para mitigar los riesgos intrínsecos y extrínsecos en este paciente. Sin embargo, es esencial reconocer las lesiones potencialmente prevenibles, diferenciando de lesiones posiblemente inevitables así como realizar un diagnóstico diferencial de las lesiones resultantes de la patogénesis del virus en sí.

DESCRIPTORES: Úlcera por Presión; COVID-19; Cuidados críticos; Heridas y Traumatismos; Enfermería.

\section{INTRODUÇÃO}

A doença causada pelo novo coronavírus, também conhecida como COVID-19, é uma enfermidade que acomete as vias respiratórias, na qual o vírus apresenta-se extremamente infeccioso e gera no individuo acometido condições clínicas variadas, bem como sintomas diversos. Sabe-se que a maioria das pessoas infectadas apresentará a forma mais leve da doença. No entanto, $14 \%$ dos doentes podem desenvolver sua forma crítica, necessitando de oxigenoterapia e, pelo menos, $5 \%$ evoluirão para uma pneumonia mais grave. Esse grupo necessita de tratamento em unidade de terapia intensiva (UTI), utilizando com frequência ventilação não invasiva (VNI) e intubação com consequente suporte em ventilação mecânica ${ }^{1}$.

Pacientes internados em UTI, intubados sob ventilação mecânica, frequentemente apresentarão alto risco de desenvolver lesões de pele, especialmente lesão por pressão (LP), devido às suas condições clínicas e hemodinâmicas comprometidas, imobilidade no leito, percepção sensorial diminuída, entre outros fatores intrínsecos e extrínsecos que favorecem o desenvolvimento dessas lesões ${ }^{2}$.
A LP é considerada como um evento adverso relacionado à saúde, sendo na maioria dos casos evitável ${ }^{3}$. Dados de uma revisão sistemática sobre prevalência e incidência de LP em UTI apontaram que a prevalência acumulada de LP ao redor do mundo variou de 16,9 a $23,8 \%^{2}$. Além disso, nota-se uma incidência de LP até dez vezes maior nos pacientes submetidos a cuidados críticos, quando comparados àqueles internados em outras unidades hospitalares ${ }^{4}$. Assim, intervenções preventivas para LP necessitam ser instituídas desde a admissão do paciente na UTI, visando diminuição das taxas de incidência e prevalência, segurança do paciente e qualidade da assistência.

Porém, a prevenção de LP no contexto do coronavírus apresenta-se como uma situação ainda mais desafiadora, pois as alterações decorrentes da infecção expõem o paciente à maior instabilidade, menor oxigenação tecidual, tempo de internação em unidade crítica prolongado e possível dificuldade de reposicionamento, fatores que favorecem o desenvolvimento das $\mathrm{LPs}^{5}$. Além disso, associam-se aos aspectos relacionados aos serviços de saúde, tais como escassez de materiais e tecnologias para prevenção, recursos humanos limitados, entre outros 5 . 
Dessa forma, com intuito de promover a segurança dos pacientes, desde o início da pandemia, sociedades internacionais como National Pressure Injury Advisory Panel (NPIAP) vêm publicando orientações gerais para a prevenção de LP. Essas publicações levam em consideração aspectos específicos do desenvolvimento de LP em pacientes com COVID-19. Dessa forma, o objetivo deste artigo é refletir sobre a prevenção de LP em pacientes com COVID-19 na terapia intensiva à luz das recomendações internacionais.

\section{MÉTODO}

Trata-se de um estudo do tipo reflexão teórica fundamentada no conceito de LP definido pelo NPIAP5

Algumas concepções balizaram este estudo, conformando o marco referencial para a compreensão da problemática. Ou seja, essas concepções subsidiaram os pressupostos e a delimitação para a abordagem do objeto em estudo, funcionando como conceitos de apoio e meios para construção desta reflexão ${ }^{6}$.

Assim, o marco referencial deste estudo foi as seguintes recomendações do NPIAP para o contexto da COVID-197:

- Pressure injury prevention - PIP tips for prone positioning;

- Best practices for the prevention of medical device-related pressure injuries;

- Skin manifestations with COVID-19: the purple skin and toes that you are seeing may not be deep tissue pressure injury;

- Unavoidable pressure injury during COVID-19 pandemic: a position paper from the National Pressure Injury Advisory Panel;

\section{LESÃO POR PRESSÃO NO CONTEXTO DA COVID-19}

Após leitura e análise dos documentos específicos para pacientes com COVID-19 publicados pelo NPIAP $^{7}$ foi possível categorizar o conteúdo em três eixos temáticos:

- Fatores de risco de desenvolvimento de LP específicos no paciente com COVID-19;

- Recomendações internacionais sobre LP em pacientes com COVID-19;

- Desafios para implementação das recomendações de prevenção de LP na pandemia de COVID-19 no cenário brasileiro.

\section{Fatores de risco de desenvolvimento de lesão por pressão específicos para o paciente com COVID-19}

Há uma série de fatores que aumentam o risco de LP em pacientes críticos ${ }^{2}$. No entanto, a infecção por COVID-19 trata-se de um agravo novo e sua relação com o desenvolvimento de LP ainda carece de estudos bem delineados. Assim, o NPIAP chama a atenção para os principais fatores intrínsecos e extrínsecos relacionados à COVID-19 que podem contribuir para maior risco de $\mathrm{LP}^{7}$.

\section{Fatores intrínsecos}

Coagulopatia sistêmica: a doença, síndrome respiratória aguda grave do coronavírus 2 (Sars-CoV-2), favorece uma coagulopatia sistêmica com hipercoagulação e oclusão microvascular, que está associada a diversas complicações clínicas e ao aparecimento de manifestações cutâneas no paciente. Embora as manifestações cutâneas apresentem-se comumente como áreas arroxeadas, elas não devem ser confundidas com LP tissular profunda, é necessário avaliar se a área em questão foi submetida a pressão e cisalhamento ${ }^{8}$. Além disso, esse estado de hipercoagulação pode favorecer o desenvolvimento de uma LP de deterioração rápida, mesmo quando em uso das medidas preventivas adequadas ${ }^{7}$. Ademais, a oclusão microvascular tem impacto em vários órgãos, o que favorece o desenvolvimento de múltiplas disfunções orgânicas, podendo ter impacto direto na manutenção da integridade da pele devido ao menor aporte de suprimento para os tecidos, aumento de líquido intersticial, com consequente aumento da vulnerabilidade da pele ${ }^{7}$.

Aspectos nutricionais: também são importantes nesse contexto, pois o paciente com COVID-19 apresenta estado hipercatabólico com maior risco de déficit nutricional, tanto pela infecção viral quanto pelas intervenções, tais como posicionamento em prona, uso de sedativos em doses altas, entre outros. É comum o desenvolvimento de diarreia, com consequente aumento da umidade da pele e dermatite associada à incontinência ${ }^{7}$.

Gravidade e instabilidade hemodinâmica: é um quadro bastante comum nesses casos, que pode limitar o reposicionamento e promover hipóxia tecidual. É preciso ressaltar que os sobreviventes a esse período de instabilidade apresentam alto risco de LP devido às consequências do 
tratamento, que podem resultar em desnutrição e debilidade com reabilitação prolongada ${ }^{7}$. Considerando a gravidade da doença e independente desses fatores específicos da COVID-19, o NPIAP reforça para a manutenção das intervenções preventivas para LP em todas as fases do tratamento ${ }^{8}$. Também chama a atenção para a realização de diagnóstico diferencial entre manifestação cutânea da COVID-19 e $\mathrm{LP}^{7,8}$, assim como a análise de causa raiz do aparecimento da LP, a fim de diferenciar lesões evitáveis de inevitáveis? ${ }^{7}$.

\section{Fatores extrínsecos}

Recursos materiais limitado/inadequado: frente ao aumento da demanda e a gravidade do paciente no contexto pandêmico é possível que alguns sistemas de saúde apresentem dificuldades em adquirir e disponibilizar os recursos adequados para prevenção. Entre eles, a superfície de suporte adequada para promover a redistribuição da pressão ${ }^{5}$.

Recursos humanos limitados: no período da pandemia pode haver escassez de profissionais no mercado, bem como o subdimensionamento da equipe multiprofissional. Ainda deve ser considerado que a redução das possibilidades de formação dos profissionais da saúde para as melhores práticas podem comprometer a implementação de medidas preventivas de LP.

\section{Recomendações internacionais sobre lesão por pressão em pacientes com COVID-19}

\section{Recomendações para prevenção de lesão por pressão no paciente submetido ao decúbito ventral}

Dentre as complicações apresentadas pelos pacientes acometidos por COVID-19, destaca-se a síndrome do desconforto respiratório agudo grave (SDRA), com uma prevalência de até $17 \%{ }^{9}$. Entre as estratégias de manejo clínico da SDRA, o posicionamento do paciente em decúbito ventral, também conhecido como prona, pode ser utilizado como adjuvante para melhora no recrutamento alveolar ${ }^{10}$. O posicionamento em prona para o tratamento de SDRA grave já era adotado como estratégia de tratamento antes da pandemia por COVID-1911 , no entanto, a prática desse posicionamento apresentou-se mais frequente nos pacientes acometidos pelo vírus. Atrelado a isso, o risco do desenvolvimento de LPs relacionadas a esse posicionamento, que já era relatado como uma das principais complicações, ganhou destaque no contexto mundial, necessitando de diretrizes e intervenções focadas para prevenção. As intervenções mais relevantes para prevenção de LP no paciente submetido a decúbito ventral são ${ }^{7,10}$ :

- Escolha adequada da superfície de suporte, de forma que promova a redistribuição de pressão, além de fazer uso de dispositivos auxiliares para o posicionamento, tais como coxins e travesseiros para alívio de pressão;

- Inspeção rigorosa da pele antes da realização da pronação, com consequente proteção de áreas expostas a maior risco, podendo ser indicada a utilização de cobertura profilática em pacientes de alto risco. Os principais pontos de pressão nesse posicionamento são: testa, bochecha, queixo, clavícula, cotovelo, inframamária, genitais, pelve, joelhos, dorso e dedos dos pés, bem como o entorno de dispositivos médicos;

- Manutenção da pele limpa e seca através da higienização adequada com produtos de limpeza com $\mathrm{pH}$ levemente ácido. Atenção especial deve ser dada aos pacientes com incontinência urinária, fecal e mista, pois a umidade é um fator de risco importante para o desenvolvimento de LP;

- Realização de pequenos reposicionamentos do paciente a cada duas ou quatro horas. Sugere-se a utilização da técnica de reposicionamento nadador, na qual alterna-se a posição dos braços e da cabeça.

\section{Recomendações para prevenção de lesão por pressão relacionada a dispositivos médicos}

Além das LPs relacionadas ao posicionamento do paciente, atenção especial deve ser dada à prevenção de lesão por pressão relacionada a dispositivos médicos (LPRDM), que pode aparecer tanto durante o posicionamento ventral quanto nos demais posicionamentos. Vale destacar que o paciente crítico, em geral, faz uso de diversos dispositivos invasivos e não invasivos, sendo, muitos deles, essenciais para sua sobrevivência, o que o expõe a maior risco de desenvolver $\mathrm{LPRDM}^{7}$. Dessa forma, faz-se necessária a implementação de intervenções específicas para prevenção, como descritas abaixo $0^{5,10}$ :

- Seleção do dispositivo de forma individualizada, considerando o tamanho adequado;

- Inspeção rigorosa da pele no local de inserção/colocação e ao redor do dispositivo;

- Monitoramento da tensão das fixações dos dispositivos e promoção do alívio de pressão, sempre que possível, além de girar e reposicionar o dispositivo regularmente, quando aplicável; 
- Evitar posicionar o paciente sobre o dispositivo médico, e em situações que não seja possível, como no caso do posicionamento em prona, deve-se verificar a possibilidade de implementação de intervenções avançadas, tais como coberturas profiláticas e/ou posicionadores e coxins específicos para prevenção;

- Orientação ao paciente, família e equipe, a fim de maximizar a adesão às medidas preventivas, bem como sinalização precoce de queixas relacionadas ao equipamento em uso;

- Utilização de cobertura profilática fina na interface dispositivo-pele, quando aplicável;

- Se possível e seguro, deve-se intercalar a utilização de máscara facial Bilevel Positive Airway Pressure (BIPAP)/ Contiunous Positive Airway Pressure (CPAP) com a utilização de cateter nasal, a fim de alternar os pontos de pressão e consequentemente diminuir o risco de LPRDM, bem como reduzir a sua gravidade quando já instalada, com atenção especial ao paciente que apresenta edema na área de aplicação do dispositivo;

- Estabelecer um rodízio dos sítios de colocação do oxímetro e dos demais dispositivos, sempre que possível.

\section{Recomendações sobre classificação de lesão por pressão inevitável em pacientes com COVID-19}

As LPs inevitáveis são definidas como lesões desenvolvidas mesmo após aplicação adequada de todas as medidas preventivas indicadas de acordo com o risco do paciente. Ou ainda naqueles casos nos quais não seja seguro a implementação das intervenções preventivas, pois a gravidade e a magnitude do risco apresentado são excessivamente altas. Cita-se como exemplo os pacientes em extrema gravidade, nos quais o mínimo reposicionamento pode gerar piora hemodinâmica ${ }^{12}$.

No contexto da COVID-19, a instabilidade clínica e hemodinâmica do paciente e aspectos relacionados à própria fisiopatologia do vírus, bem como a mudança brusca nas estruturas dos serviços de saúde fizeram com que o NPIAP se posicionasse sobre como diferenciar se a LP foi inevitável em pacientes complexos acometidos durante a pandemia ${ }^{7}$.

Dessa forma, o NPIAP reforça que o mero diagnóstico de COVID-19 não determina que a LP seja inevitável, para isso recomenda-se a observação dos seguintes aspectos ${ }^{7}$ :

- Realizar análise de causa raiz da LP, considerando fatores extrínsecos e intrínsecos ao paciente e ao serviço de saúde;
- Realizar diagnóstico diferencial entre manifestações cutâneas da COVID-19 e LP tissular profunda;

- Considerar que lesões em áreas não submetidas à pressão, cisalhamento e dispositivos provavelmente não são LP;

- Garantir que todas as medidas preventivas tenham sido executadas e registradas nos prontuários dos pacientes. Nesse caso, mesmo que o paciente desenvolva LP esta poderá ser considerada inevitável, uma vez que todas as intervenções foram realizadas adequadamente e devido à magnitude e gravidade o paciente desenvolveu LP.

\section{Desafios para implementação das recomendações de prevenção de lesão por pressão na pandemia de COVID-19 no cenário brasileiro}

No Brasil, os enfermeiros são responsáveis pela avaliação de risco de LP desde a admissão do paciente e durante toda a sua internação, além de prescrever os cuidados de prevenção e tratamento. Por estarem na linha de frente do enfrentamento à pandemia, esses sofrem com uma enorme carga de trabalho, esgotamento e fadiga a longo prazo, ameaça de infecção, risco de desenvolvimento de lesões de pele relacionadas ao uso de equipamento de proteção individual ${ }^{13} \mathrm{e}$ frustração com a morte dos pacientes a quem cuidam ${ }^{14}$. Assim, se não houver dimensionamento adequado há o risco de cuidados serem negligenciados e a segurança do paciente pode ser afetada.

O Conselho Federal de Enfermagem (COFEN) estabeleceu parâmetros mínimos de profissionais de enfermagem para atendimento aos pacientes acometidos por COVID-19 e recomendou, ainda, que o Índice de Segurança Técnica seja de $20 \%$, em razão do expressivo aumento do número de afastamento dos profissionais de enfermagem acometidos por COVID-19 ${ }^{15}$. Contudo, mesmo com a recomendação do COFEN para um dimensionamento seguro e específico em áreas de atendimento a pacientes com COVID-19, o que majora no país é o dimensionamento da Agência Nacional de Vigilância Sanitária (ANVISA), de acordo com a Resolução da Diretoria Colegiada (RDC) $\mathrm{n}^{\circ} 7^{16}$, que determina um número menor de profissionais ao ser comparada com o parecer normativo do COFEN ${ }^{15}$.

O não cumprimento do Índice de Segurança Técnica, diante de expressivo número de afastamentos de profissionais de enfermagem no país, com 23.376 afastamentos por COVID-19, de acordo com o observatório de enfermagem do 
COFEN, pode comprometer gravemente tanto o posicionamento quanto o reposicionamento do paciente, inspeção sistemática da pele, análise do processo de desenvolvimento da lesão e demais medidas preventivas que precisam ser implementadas para a prevenção de LP no contexto da COVID-19.

O subdimensionamento e falta de treinamento também podem prejudicar a notificação da LP no país. De acordo com a ANVISA, as LPs do estágio 3 em diante são de notificação obrigatória e faz-se necessária a análise causa raiz do evento. Contudo, de acordo com o documento de incidentes relacionados à assistência à saúde, com os resultados das notificações realizadas através do Sistema de Notificação para a Vigilância Sanitária (NOTIVISA), no Brasil, de junho de 2019 a maio de 2020, foram reportados 2.491 LPs estágio 3 e 792 estágio $4^{3}$. Se esses números não refletem a realidade da incidência de LP no país antes da pandemia, acredita-se que durante a pandemia, por todas as questões já mencionadas, a subnotificação e falta de análise dos eventos sejam alarmantes.

Além disso, no Brasil, enfrentam-se dificuldades relacionadas à disponibilidade de recursos materiais adequados para prevenção de eventos adversos ${ }^{1}$. Sabe-se que muitas instituições no país carecem de materiais básicos para manutenção da integridade da pele mesmo antes da pandemia, no entanto nesse período pandêmico esse problema pode ser ainda mais complexo, uma vez que a expansão do número de leitos de UTI, bem como a criação de hospitais de campanha podem não ter sido contemplados com materiais adequados, conforme as recomendações nacionais e internacionais.

\section{CONSIDERAÇÕES FINAIS}

A pandemia de COVID-19 instituiu no Brasil e no mundo um "novo normal", no qual todos terão que se adaptar e criar estratégias de enfrentamento. No quesito prevenção de LP é fundamental que os profissionais de saúde, especialmente a equipe de enfermagem, sejam capazes de implementar intervenções avançadas, de forma a mitigar os riscos intrínsecos e extrínsecos nesse paciente. No entanto, é imprescindível que sejam capazes de reconhecer as lesões potencialmente evitáveis, bem como a realização de diagnóstico diferencial de lesões decorrentes da própria patogenia do vírus.

Desafios no que tange ao subdimensionamento, à formação de profissionais com expertises em prevenção e tratamento de feridas, bem como treinamentos das equipes ficaram evidentes na pandemia, demonstrando a necessidade de organização dos serviços de saúde para assistência dos novos casos de COVID-19, bem como possíveis outras situações complexas como essa.

Esta publicação possibilitará maior acesso à informação atualizada do NPIAP pelos profissionais de saúde envolvidos na prevenção e tratamento de LP, especialmente no contexto brasileiro. Dessa forma, a prestação da assistência clínica e novos trabalhos de pesquisa poderão ser orientados por meio dessas reflexões.

Destaca-se como limitação do estudo a escassez de publicações específicas sobre prevenção de LP no contexto pandêmico de COVID-19, bem como a emergência diária de novas publicações sobre a patogenia do vírus em questão.

\section{CONTRIBUIÇÃO DOS AUTORES}

Conceitualização: Ramalho AO; Metodologia: Moraes JT; Redação - Primeira Versão: Ramalho AO e Paula Freitas; Redação - Revisão \& Edição: Ramalho AO, Paula Freitas, Moraes JT e Nogueira PC; Supervisão: Nogueira PC e Moraes JT.

\section{REFERÊNCIAS}

1. Ministério da Saúde (BR). Orientações para manejo de pacientes com COVID-19. Brasília (DF): Ministério da Saúde; 2020.

2. Chaboyer WP, Thalib L, Harbeck EL, Coyer FM, Blot S, Bull CF et al. Incidence and prevalence of pressure injuries in adult intensive care patients: a systematic review and meta- analysis. Crit Care Med 2018 Nov;46(11):e1074-81. https:// doi.org/10.1097/CCM.0000000000003366

3. Agência Nacional de Vigilância Sanitária (BR). Nota Técnica GVIMS/GGTES N03/2017. Práticas seguras para prevenção de lesão por pressão em serviços de saúde. [Internet]; 2017 [citado 21 abr 2020]. Disponível em: https://www20.anvisa. 
gov.br/segurancadopaciente/index.php/alertas/item/notatecnica-gvims-ggtes-03-2017

4. Nowicki JL, Mullany D, Spooner A, Nowicki TA, Mckay PM, Corley A et al. Are pressure injuries related to skin failure in critically ill patients? Aust Crit Care 2018 Sep;31(5):257-63. https://doi.org/10.1016/j.aucc.2017.07.004

5. European Pressure Ulcer Advisory Panel, National Pressure Injury Advisory Panel, Pan Pacific Pressure Injury Alliance. Prevention and treatment of pressure ulcers/injuries: quick reference guide. $3^{\text {a }}$ ed. EPUAP/NPIAP/PPPIA; 2019.

6. Pessalacia JDR, Zoboli ELCP, Ribeiro IK. Equidade no acesso aos cuidados paliativos na atenção primária à saúde: uma reflexão teórica. R Enferm Cent O Min 2016 jan/abr; [citado 2020 out 14]; 1(6):2119-39. Disponível em: http://www.seer. ufsj.edu.br/index.php/recom/article/view/1072

7. National Pressure Injury Advisory Panel. Unavoidable pressure injury during COVID-19 pandemic: a position paper from the National Pressure Injury Advisory Panel 2020.

8. Black JM, Berke CT. Deep tissue pressure injury: a clinical perspective regarding a condition that evolves under the skin. Innovations and Emerging Technologies in Wound Care 2020. 171-81. https://doi.org/10.1016/B978-0-12815028-3.00010-9

9. Chen N, Zhou M, Dong X, Qu J, Gong F, Han Y et al. Epidemiological and clinical characteristics of 99 cases of 2019 novel coronavirus pneumonia in Wuhan, China: a descriptive study. Lancet 2020;395(10223):507-13. https:// doi.org/10.1016/S0140-6736(20)30211-7
10. Moore Z, Patton D, Avsar P, McEvoy NL, Curley G, Budri $A$ et al. Prevention of pressure ulcers among individuals cared for in the prone position: lessons for the COVID-19 emergency. J Wound Care 2020 Jun;29(6):312-20. https:// doi.org/10.12968/jowc.2020.29.6.312

11. Guérin C. Prone positioning acute respiratory distress syndrome patients. Ann Transl Med 2017 Jul;5(14):289. https://doi.org/10.21037/atm.2017.06.63

12. Edsberg LE, Langemo D, Baharestani MM, Posthauer ME, Goldberg M. Unavoidable pressure injury: state of the science and consensus outcomes. J Wound Ostomy Continence Nurs 2014 Jul/Aug;41(4):313-34. https://doi. org/10.1097/WON.0000000000000050

13. Ramalho AO, Freitas PSS, Nogueira PC. Medical devicerelated pressure injury in health care professionals in times of pandemic. ESTIMA Braz J Enterostomal Ther 2020;18:e0120. https://doi.org/10.30886/estima.v18.867_PT

14. Shen X, Zou X, Zhong X, Yan J, Li L. Psychological stress of ICU nurses in the time of COVID-19. Crit Care 2020 May;24(1):200. https://doi.org/10.1186/s13054-020-02926-2

15. Conselho Federal de Enfermagem. Parecer normativo n 002/2020 - Exclusivo para vigência da pandemia COVID-19. Brasília (DF); 2020.

16. Resolução - RDC ANVISA n. 07, de 24 de fevereiro de 2010. Dispõe sobre os requisitos mínimos para funcionamento de Unidades de Terapia Intensiva e dá outras providências. Diário Oficial da União [periódico na internet], Brasília (DF). 25 fev 2010 [citado 13 out 2020]. Disponível em: https:// www20.anvisa.gov.br/segurancadopaciente/index.php/ legislacao/item/rdc-07-2010 